\title{
Research Paper \\ Prevalence and Determinants of Eczema Among Elementary School Children, Southeast of Iran, 2019
}

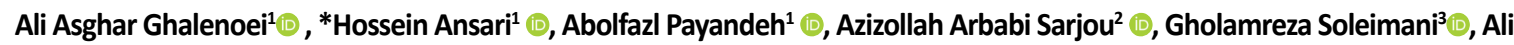 \\ Meshkinian ${ }^{4}$ (1)
}

1. Department of Epidemiology and Biostatistics, Health Promotion Research Center, Zahedan University of Medical Sciences, Zahedan, Iran

2. Department of Nursing, Community Nursing Research Center, Zahedan University of Medical Sciences, Zahedan, Iran.

3. Department of Pediatrics, Children and Adolescents Health Research Center, School of Medicine, Zahedan University of Medical Sciences, Zahedan, Iran.

4. Department of Occupational Health Engineering, Health Promotion Research Center, Zahedan University of Medical Sciences, Zahedan, Iran

\begin{tabular}{|c|c|}
\hline $\begin{array}{l}\text { Use yourdevice to tsan } \\
\text { and read the article online }\end{array}$ & dtation: Ghalenoei AA, Ansari H, Payandeh A, Arbabi Sarjou A, Soleimani Gh, Meshkinian A. [Prevalence and Determi- \\
\hline 口PAB & nants of Eczema Among Elementary School Children, Southeast of Iran, 2019 (Persian)]. Journal of Arak University of Medical \\
\hline Fiftis & Sciences (JAMS). 2021; 24(3):438-449. https://doi.org/10.32598/JAMS.24.3.6370.1 \\
\hline a & del'https://doi.org/10.32598/JAMS.24.3.6370.1 \\
\hline
\end{tabular}

Key words:

Eczema, Dermatitis, Allergy, Children, ISAAC

\section{A B STRACT}

Article Info:

Received: 25 Nov 2020 Accepted: 08 Aug 2021 Available Online: 01 Aug 2021
Background and Aim Genetic and environmental factors are known to be related to the development of childhood Eczema. We aimed to assess the ecological factors associated with the prevalence of Eczema among children using the ISAAC questionnaire.

Methods \& Materials A total of 1600 primary school girls and boys were selected and examined by multistage random sampling in this cross-sectional study. Data were collected and completed using the International ISAAC Questionnaire, including demographic questions and risk factors through interviews with parents, especially the student's mother. The data were analyzed in SPSS19 software using the Chi-square test and multiple logistic regression.

Ethical Considerations This study was approved by the Ethics Committee of Zahedan University of Medical Sciences (Code: REC.1397.481).

Results The prevalence of current Eczema was 10.4\% (CI95\% 8.9-11.9). Co-morbidity with Asthma and Rhinitis was $82.8 \%$ and $74.5 \%$, respectively. In multiple logistic regression models, history of allergies in family members (OR: $2.35 \mathrm{Cl}$ (95\%:1.53-3.59), father snoring (OR: $3.00 \mathrm{CI}$ \%5:1.98-4.55), Keeping any animal in (OR: 1.6 Cl95\%:1.002-2.58), family size (OR:0.85 Cl95\%:0.68-0.95), exposure to tobacco smoke at home (OR:2.84 Cl95\%:1.18-6.81), showed a significant relationship with the occurrence of students' Eczema.

Conclusion Exposure to tobacco smoke at home, keeping any animal at home are effective factors in the prevalence of Eczema in children. By eliminating these factors, the prevalence of this disease can be reduced. However, the mothers have the most crucial role in screening and early diagnosis of Eczema in school children regarding determinants in this study. According to the results of this study, it is suggested that parents' education, especially mothers, be emphasized by paying attention to these favorable factors.

\section{* Corresponding Author:}

Hossein Ansari, PhD

Address: Dr. Hesabi Square, University of Medical Sciences, School of Health, Department of Epidemiology and Statistics.

Tel: +98 (912) 6309480

E-mail: ansarih88@gmail.com 


\section{Extended Abstract}

\section{Introduction}

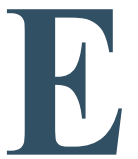

czema is a chronic inflammatory disease of the skin characterized by an itchy red rash known as "atopic eczema." Health professionals have struggled with Eczema for a long time, but the exact cause and appropriate treatment have not yet been determined [1]. Eczema harms the quality of life of affected children and their families [2] and can disrupt the social life of these children, especially in the school environment [1]. Eczema is positively associated with urban and industrial environments, obesity, higher socioeconomic status, lower family size [1], environmental factors, and genetic factors play an essential role in disease progression
[5]. This study aimed to evaluate the relationship between students' Eczema and a range of environmental factors using the International Pediatric Asthma and Allergy Questionnaire (ISAAC) to prevent its complications to some extent by timely diagnosis and management of these factors.

\section{Materials and Methods}

This cross-sectional study was performed on an elementary school student in the 2019 academic year. Sampling was based on a multistage sampling of dual areas of education in proportion to the volume of each floor (8 floors). Since the prevalence of asthma and allergic symptoms, by $3.5 \%$ and considering the value of $\mathrm{d}=0.01$ and alpha 0.05 . and considering the effect of the design equal to 1.2 , the final sample size was estimated to be about 1600 people [13].

Table 1. Analysis of eczema-related questions in students by gender

\begin{tabular}{ccc}
\hline & Questions \\
\hline Gender & Boys $(n=830) /$ Girls $(n=770) /$ Total $(\mathrm{N}=1600)$ & $P$ (Pearson Chi-square) \\
\hline
\end{tabular}

Has your child ever had an itchy rash that appears and disappears for at least 6 months?

\begin{tabular}{|c|c|c|c|c|}
\hline Yes & $126(15.2)$ & 75(9.7) & 201(12.6) & 0.001 \\
\hline \multicolumn{5}{|c|}{ Has your child had an itchy rash in the last 12 months?* } \\
\hline Yes & $101(12.2)$ & $65(88.4)$ & 166(10.4) & 0.23 \\
\hline \multicolumn{5}{|c|}{$\begin{array}{c}\text { Has this itchy rash affected any of the following points at any time: wrinkles on the elbows, behind the knees, in front of the ankles } \\
\text { around the neck, ears, and the eyes? }\end{array}$} \\
\hline Yes & $140(16.8)$ & $65(8.4)$ & 205(12.8) & 0.47 \\
\hline \multicolumn{5}{|c|}{ At what age did your child first develop an itchy rash? } \\
\hline & $4(0.5)$ & $5(0.6)$ & $9(0.6)$ & \\
\hline & $29(3.5)$ & $20(2.6)$ & $49(3.1)$ & 0.52 \\
\hline & $68(8.2)$ & $40(5.2)$ & $108(6.8)$ & \\
\hline \multicolumn{5}{|c|}{ Has the rash been completely clear in the last 12 months? } \\
\hline Yes & $85(10.2)$ & $51(6.6)$ & $136(8.5)$ & 0.35 \\
\hline \multicolumn{5}{|c|}{ How many times in the last 12 months has your child waked up at night because of this rash? } \\
\hline & $70(8.4)$ & $43(5.6)$ & $113(7.1)$ & \\
\hline & $23(2.8)$ & 15(1.9) & $38(2.4)$ & 0.81 \\
\hline & $8(1.0)$ & $7(0.9)$ & $15(0.9)$ & \\
\hline \multicolumn{5}{|c|}{ Has your child ever had Eczema diagnosed by a doctor? } \\
\hline Yes & $56(6.7)$ & $55(7.1)$ & $111(6.9)$ & 00.75 \\
\hline
\end{tabular}

* Because some participants have expressed more than one conflict area, the number has risen to more than 166. 
Table 2. Coefficients for eczema-related independent variables in students in multiple logistic regression model

\begin{tabular}{|c|c|c|c|c|c|}
\hline \multicolumn{2}{|c|}{ Independent Variables } & \multirow{2}{*}{$\begin{array}{c}\begin{array}{c}\text { Crude Odds } \\
\text { Ratio (CI) }\end{array} \\
2.5(1.66-3.76) \\
\end{array}$} & \multirow{2}{*}{$\begin{array}{c}\mathbf{P} \\
0.0001\end{array}$} & \multirow{2}{*}{$\begin{array}{c}\begin{array}{c}\text { Adjusted Odds } \\
\text { Ratio (Cl) }\end{array} \\
2.35(1.53-3.59) \\
\end{array}$} & \multirow{2}{*}{$\begin{array}{c}\mathbf{P} \\
0.0001\end{array}$} \\
\hline Family history of allergies & Has it & & & & \\
\hline Does not have & 1 & & 1 & & \\
\hline Snoring father & Has it & $3.44(2.31-5.14)$ & 0.0001 & $3.00(1.98-4.55)$ & 0.0001 \\
\hline Does not have & 1 & & 1 & & \\
\hline Keeping a pet at home & Has it & $1.98(1.24-3.07)$ & 0.002 & $1.60(1.002-2.58)$ & 0.04 \\
\hline Does not have & 1 & & 1 & & \\
\hline Family dimension & Slightly & $0.86(0.73-1.02)$ & 0.08 & $0.85(0.68-0.95)$ & 0.01 \\
\hline \multirow{4}{*}{ Smoking } & lways) & $1.44(0.18-11.30)$ & 0.72 & $1.14(0.31-9.63)$ & 0.9 \\
\hline & ften) & $3.73(1.67-8.32)$ & 0.001 & $2.84(1.18-6.81)$ & 0.01 \\
\hline & ometimes) & $2.37(1.39-4.03)$ & 0.001 & $1.81(1.04-3.17)$ & 0.03 \\
\hline & & 1 & & 1 & \\
\hline
\end{tabular}

The data collection tool was a questionnaire established by the International Asthma and Allergy Study in Children (ISAAC) in 1992 and proposed to different countries [12].

The questionnaires were revised to minimize incomplete data. Also, the intended variable that had lost data was imputation by regression method before analyzing the data. Data were described and presented using descriptive statistics, including tables, frequencies, percentages, and chi-square test. To investigate the simultaneous effect of independent variables on the incidence of Eczema using the multiple logistic regression method, variables whose significance level was less than 0.2 in univariate analysis by chi-square test method were entered into the model. Data in SPSS v. 19 were analyzed with a significance level of 05.0.

\section{Results}

In terms of sex distribution, 830 boys (51.9\%) and 770 girls $(48.1 \%)$ were included in the study. The prevalence of skin problems based on the Isaac questionnaire is shown in Table 1 in two groups. The prevalence of itchy rash during life in boys and girls $(95 \% \mathrm{CI}=12.8-17.6) 15.2 \%$ and $(95 \% \mathrm{CI}=7.8-11.9) 9.7 \%$, respectively, which is statistically significant and had a higher prevalence in males $(\mathrm{P}=0.001)$. Also, the prevalence of itchy rash in the last 12 months (second question) in boys and girls (95\% $\mathrm{CI}=10.0-14.5)$ was $12.2 \%$ and $(95 \% \mathrm{CI}=6.5-10.5) 8.4 \%$, respectively this difference was not statistically significant $(\mathrm{P}=0.2)$. The overall prevalence of Eczema diagnosed by a physician $(95 \% \mathrm{CI}=5.7-8.1 \%$ ) was $6.9 \%$ (Table 1$)$.

There was a strong association with other allergic diseases: $82.8 \%$ of students with current Eczema had current asthma, and $74.5 \%$ had current rhinitis (Table 1). In univariate analysis, there is a history of allergy in the family $\mathrm{OR}=2.5$, father snoring $\mathrm{OR}=3.4$, maternal pregnancy over the age of $35 \mathrm{OR}=1.3$, household dimension $\mathrm{OR}=0.5$, keeping a pet at home $\mathrm{OR}=1.9$, and smoking once a week at home $\mathrm{OR}=3.7$ showed a significant relationship with students' Eczema $(\mathrm{P}<0.05)$. In the final logistic regression model, the variables of family history of allergy were $\mathrm{OR}=2.35$, smoking once a week at home $\mathrm{OR}=2.84$, household dimension $\mathrm{OR}=0.85$, keeping a pet at home $\mathrm{OR}=1.60$, and history of father snoring $\mathrm{OR}=3.00$ showed a significant relationship with the prevalence of Eczema in Zahedan city students (Table 2).

\section{Discussion and Conclusion}

The prevalence of eczema symptoms during life and the last 12 months in Zahedan city students was $12.6 \%$ and $10.4 \%$, respectively. The frequency of these symptoms in Ahwaz City students was 8.4 and 5.3\% [12] and in the Turkish study was $7.5 \%$ and $6.5 \%$ [15]. Eczema diagnosed by a physician in our study was $6.9 \%$. In a survey conducted by Fei Li et al. in the cities of China, the prevalence of Eczema was $4.6 \%$ [11], which is consistent with this study's results. In contrast, in the Assaluyeh study, $13 \%$ [7] 
reported that these differences might be due to environmental factors, lifestyle, or racial differences [19].

A dose-response relationship effect was observed between smoking and Eczema. Smoking at least once a week increased the risk by 2.8 -fold. Smoking has been shown to increase the risk of Eczema in studies by Jam in Bushehr city [10] and Edwin in the United States [19]. Our study showed that exposure to pets is associated with Eczema and increases the chances of getting it. However, it is known its effect varies depending on the type and amount of exposure [5]. The study of Mari Sasaki et al. showed keeping a pet during infancy increases the risk of Eczema. The protective effect of siblings has been well known since the 1990s and supports the so-called "health" hypothesis [5]. Our analysis confirmed these results, which suggest a link between a reduced chance of developing Eczema and an increased household size. Sasaki et al. [5] showed a negative relationship between the family dimension and Eczema, but a study in Bushehr city did not observe any significant association [21].

This study showed a relationship between demographic and environmental factors with Eczema and its symptoms in Zahedan city. Smoking and keeping a pet at home was one of the influential factors in the prevalence of Eczema in students, which by eliminating it, the prevalence of this disease can be reduced to some extent.

\section{Ethical Considerations}

\section{Compliance with ethical guidelines}

This study was registered with the ethics code IR.ZAUMS.REC.2018.481 in the research Ethics Committee of Zahedan University of Medical Sciences. Before the student entered this study, parents and students were informed about the goals of the project in an understandable language, and parents' consent was obtained freely and consciously. The principle of confidentiality and protection of student secrets and appropriate measures were taken to prevent its publication. In order to obtain informed consent to participate in the research, a consent form was provided to the parents or guardians of the student and their signatures were taken.

\section{Funding}

This study was sipported by Zahedan University of Medical Sciences. Also, this study was extracted from the MSc thesis of the first author at the Department of Epidemiology and Biostatistics, Health Promotion Research Center, Zahedan University of Medical Sciences, Zahedan.

\section{Authors' contributions}

Research design, data collection, data refining, results in report and discussion: Ali Asghar Ghale'e Noeie and Hossein Ansari; Data analysis: Abolfazl Payende; Preparing the final report and writing the article: Azizaleh Arbabi Serjo; Interpretation of questionnaires and patient diagnosis: Gholamreza Soleimani; Preparing a questionnaire and collecting and reviewing the collected data: Ali Meshkinian.

\section{Conflicts of interest}

The authors declared no conflict of interest.

\section{Acknowledgements}

We sincerely thank the Vice-Chancellor for Research of Zahedan University of Medical Sciences, who sponsored the project. 
This Page Intentionally Left Blank 


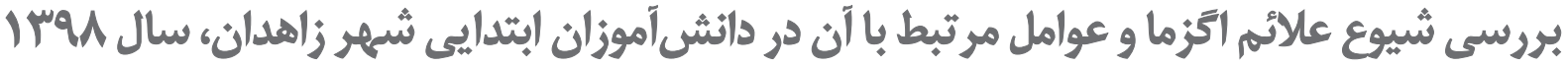

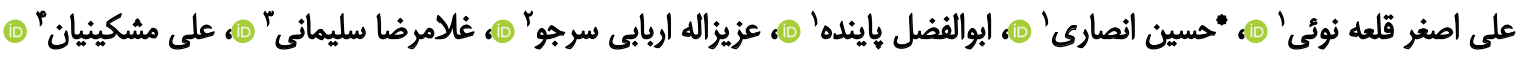

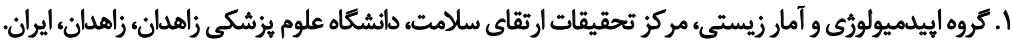

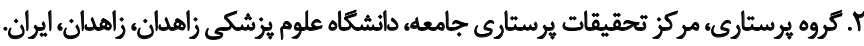

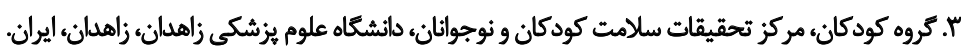

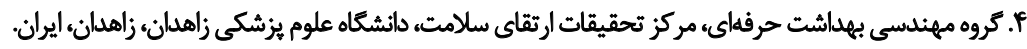

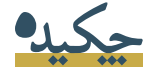

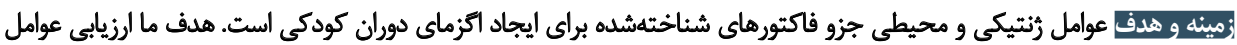

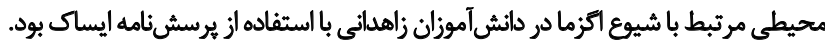

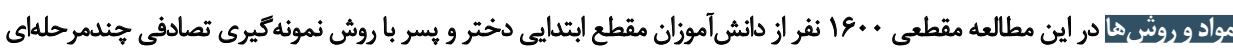

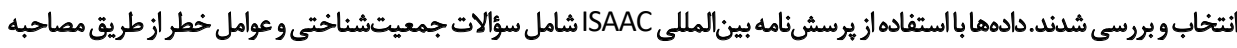

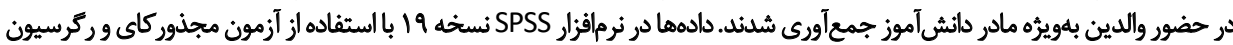
لوجستيك جيندكانه تجزيه و تحليل شد.

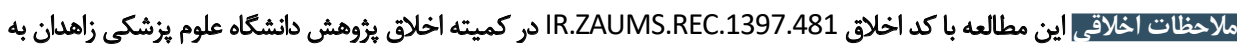
ثبت رسيد.

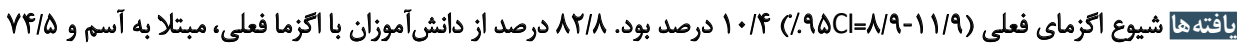

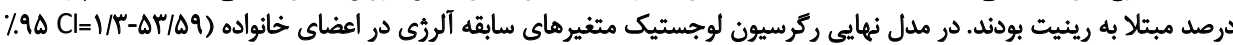

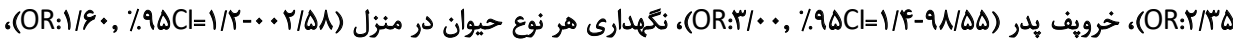

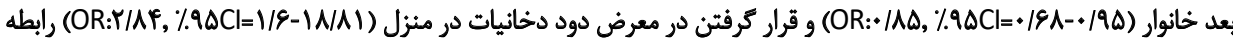

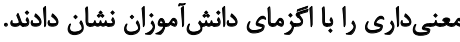

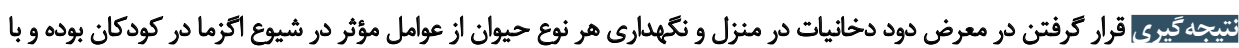

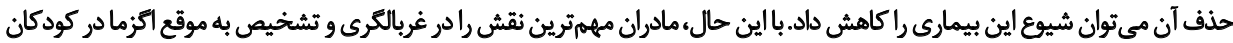

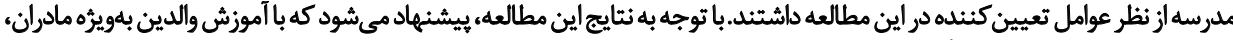
بر توجه به اين عوامل مطلوب تأكيد شود.
اطلاعات مقاله:

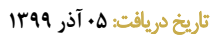

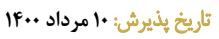

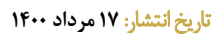

كليدواروها:

امكزماء درماتيته آلرئى، كودكان، ايساك دماتيت الرعان

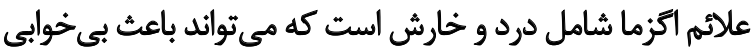

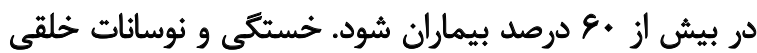

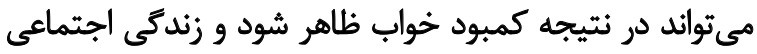

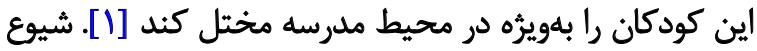

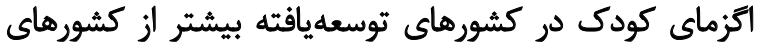

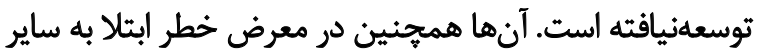

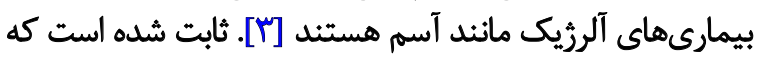

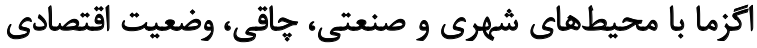

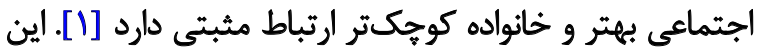

اكزما يك بيمارى التهابى مزمن يوستى است كه با بثورات

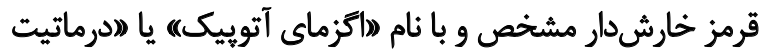

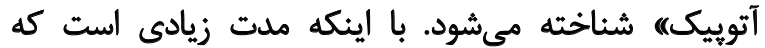

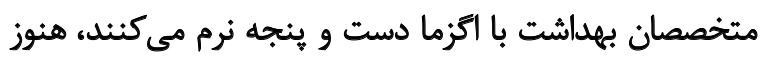

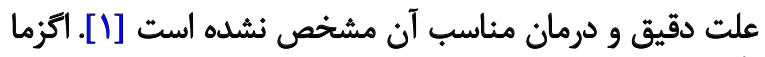

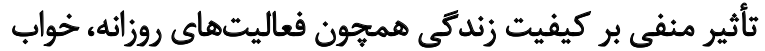

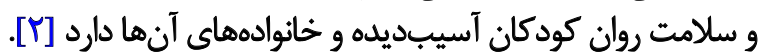


نمونه لازم از هر طبقه متناسب باجمعيت آن طبقه محاسبه شده

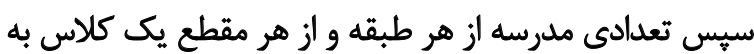

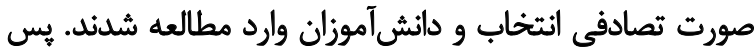

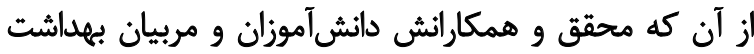

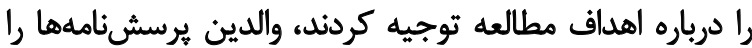

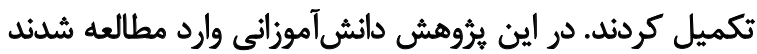

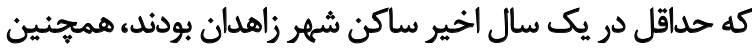

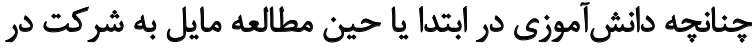

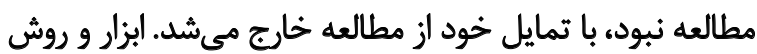

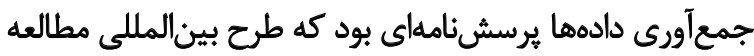

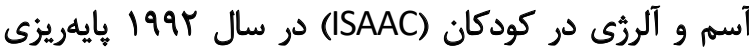

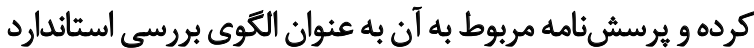

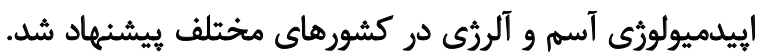

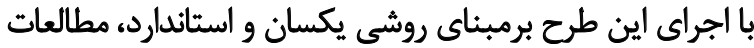

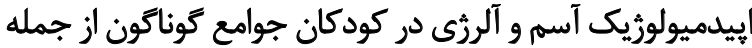

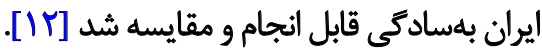

براي به حداقل رساندن دادههاى ناقص، يرسشنامهنها مجدد

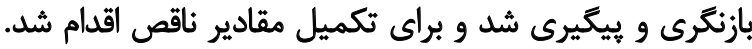

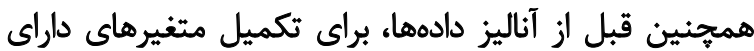

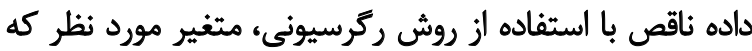

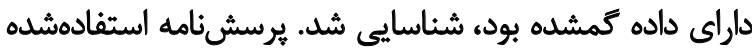

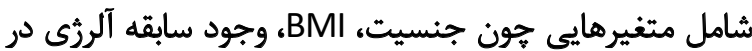

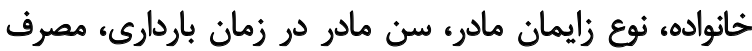

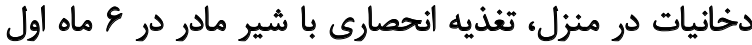

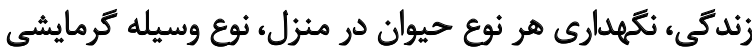

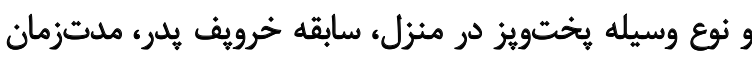

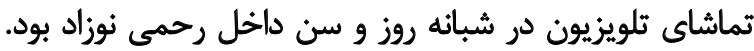

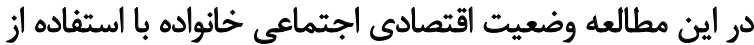

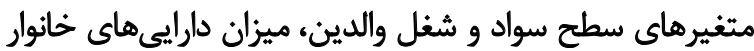

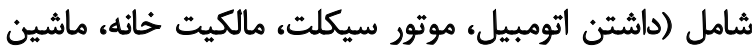

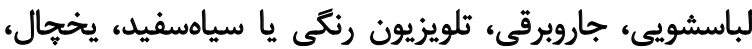

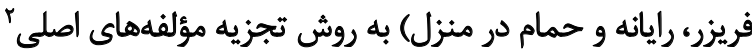

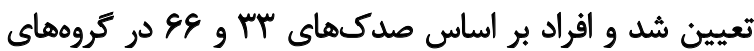

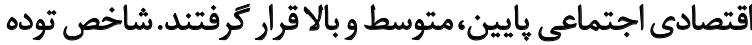
بدنى با استفاده از جدول BMI براي سن دختئي ساله سازمان جهانى بهداشت محاسبه شد (Z-scores).

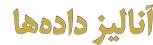

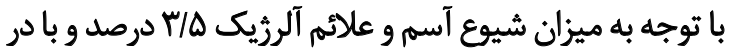

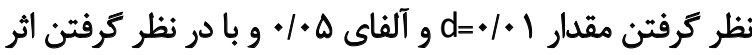

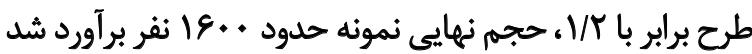

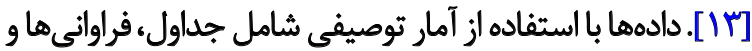

نظريه با فرضيه بهلاشت مطابقت دارد كه بيان مي كنيد قرار

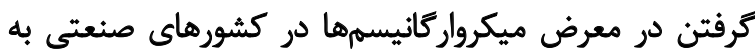

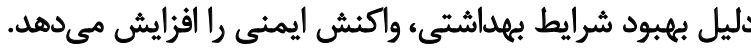

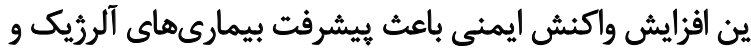

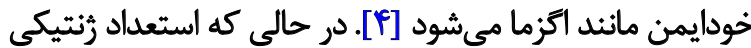

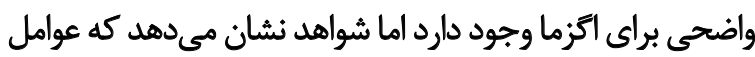

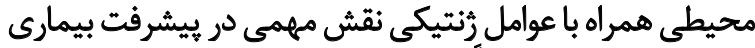

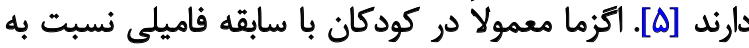

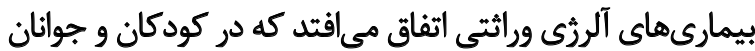

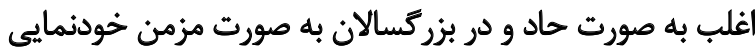

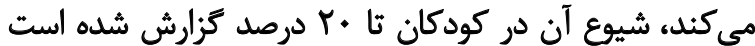

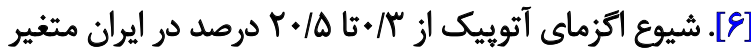

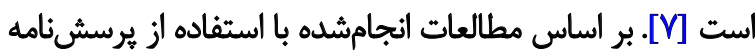

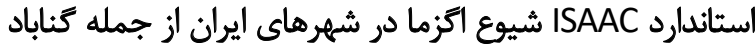

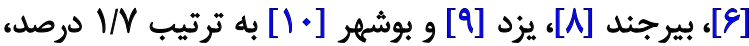

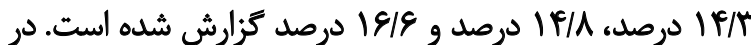

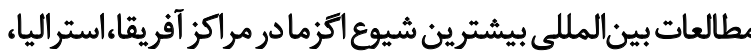

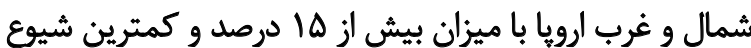

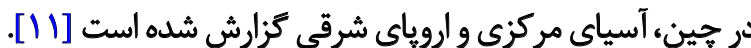
اكرجه اكزما و علاثم آن در كودكان ممكن است كشنده نباشدي،

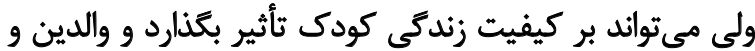

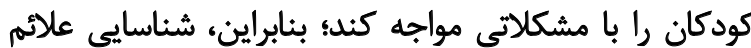
آلرزى و راههاى بيش

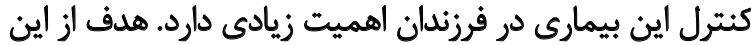

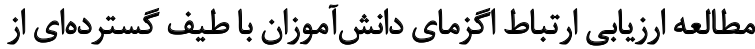

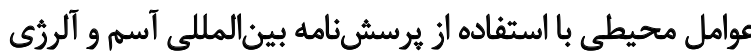

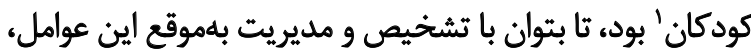

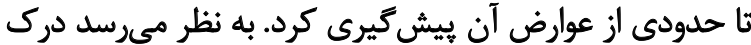

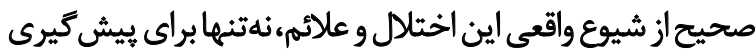

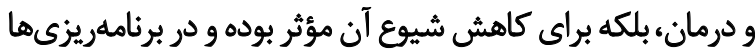

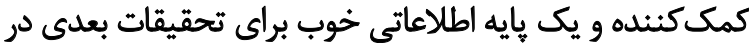

$$
\text { منوب شرق ايران باشد. }
$$

در اين مطالعه مشاهدهاى مقطعى (توصيفى تحليلى) تعداد

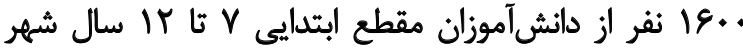

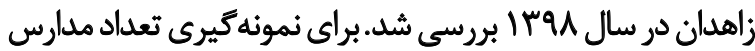

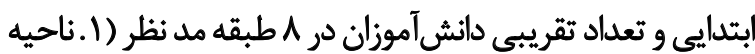

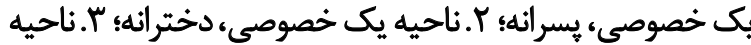

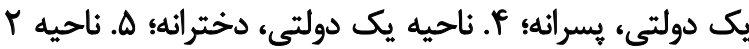

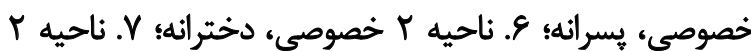

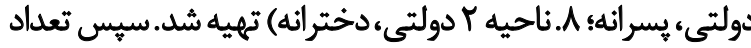


و و سابقه خرويف در يدر خانواده . •OR=1/9.

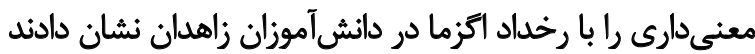

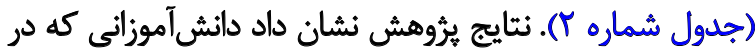

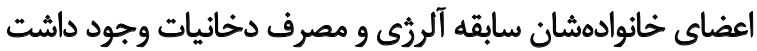

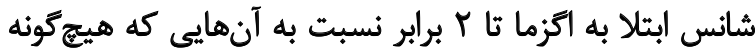

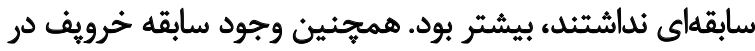

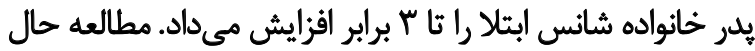

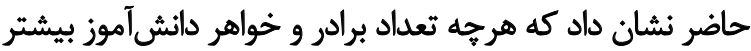

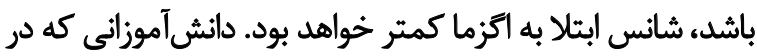

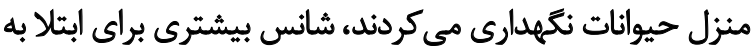
اكزما داشتند (تصوير شماره ()).

بحث

اين مطالعه با هدف شناسايى عوامل خطر براى علائم اكزما

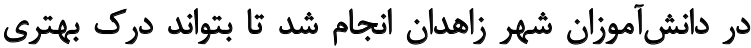

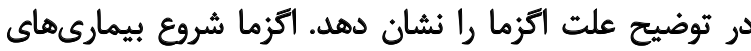
حساسيتى در كودكان است كه با بيشرفت بيت بيماري، حسيت حساسيت و ساير بيمارى جهاى حساسيتى نظير آلرثى بينى و آسم نيز إيجاد

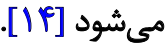

شيوع علاثم اتزما و راشهاى مزمن در طول عمر دانشآموزان

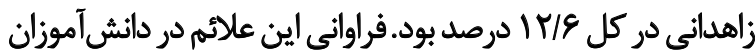

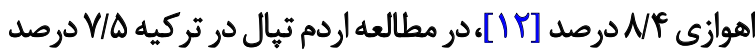

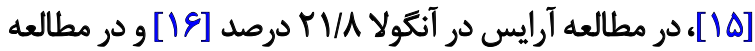

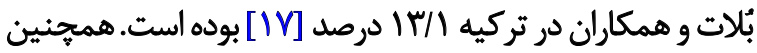

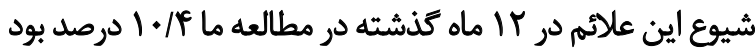

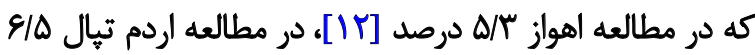

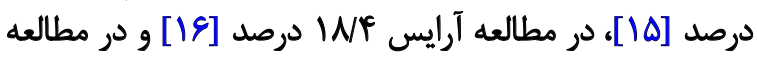

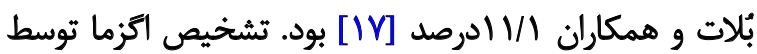

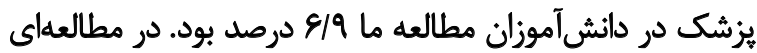

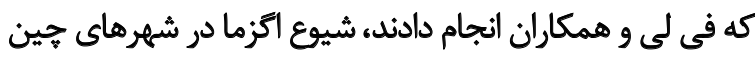

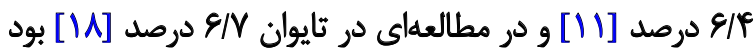

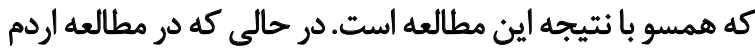

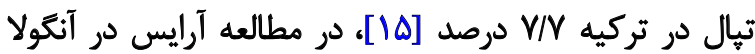

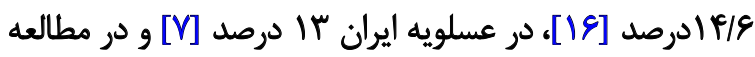

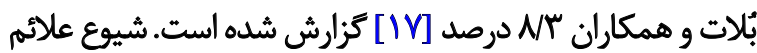

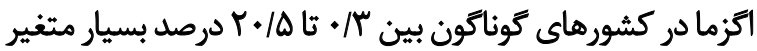

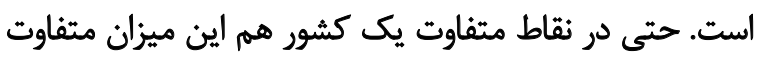

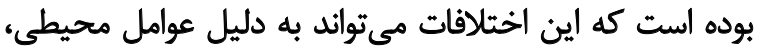

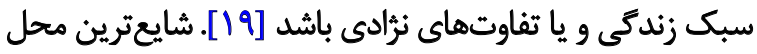

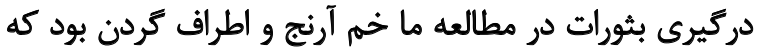

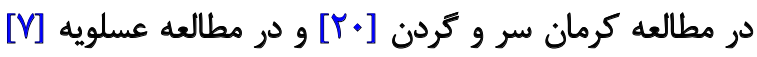

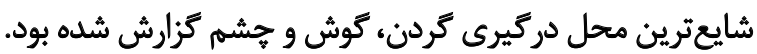

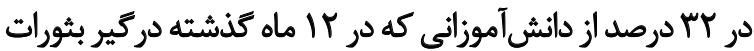

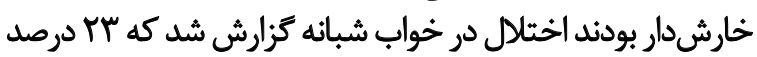

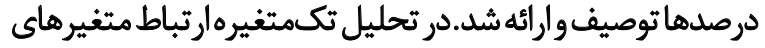

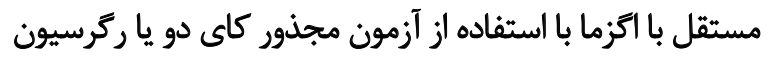

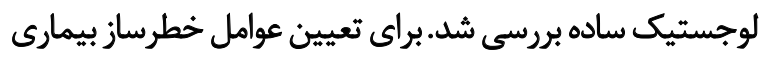

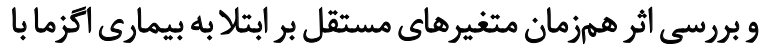

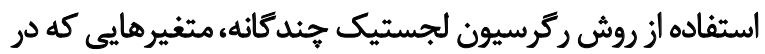

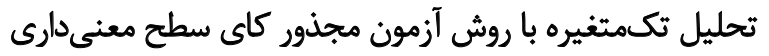

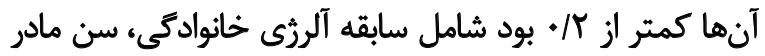

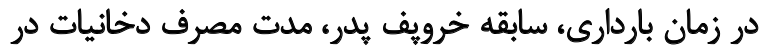

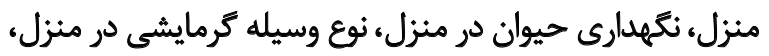

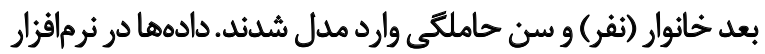

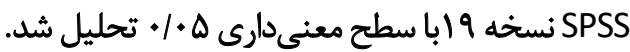

يافتهها

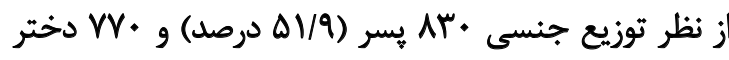

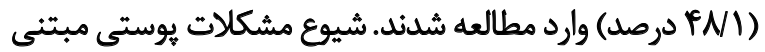

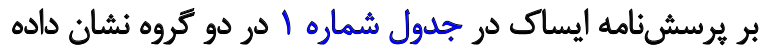

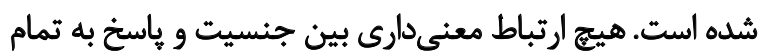

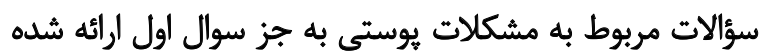

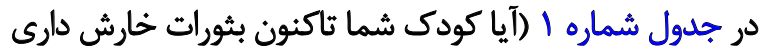

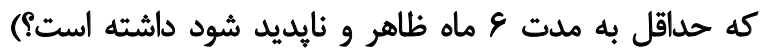

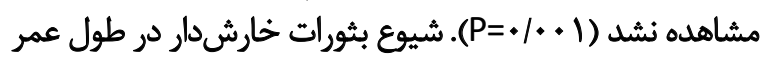

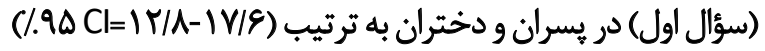

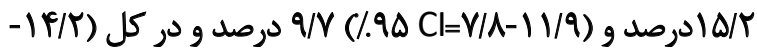

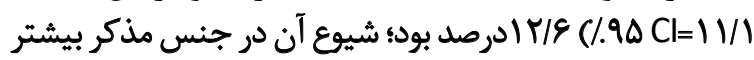

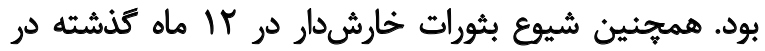

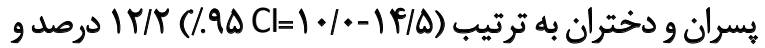

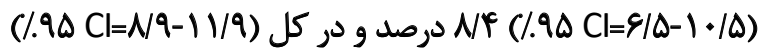

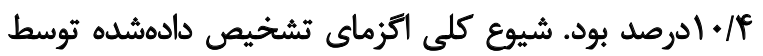

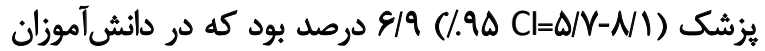

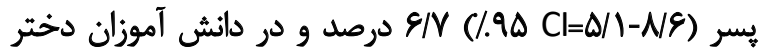

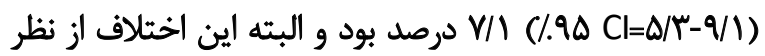

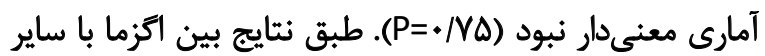

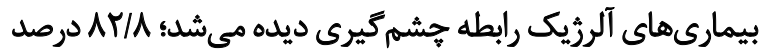

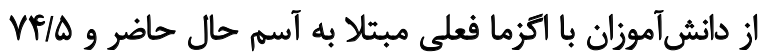

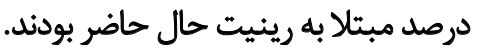

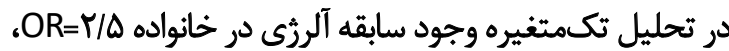

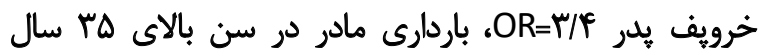

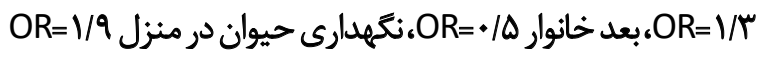

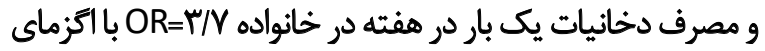

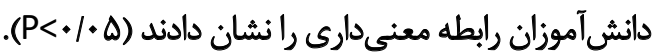

در مدل نهايى ركرسيون لجستيك متغيرهاى وجود سابقه

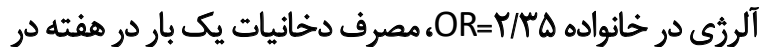

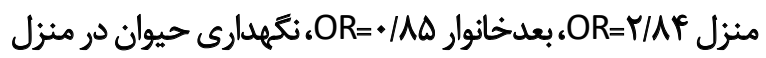


جدول 1. آناليز سؤالات مرتبط با اتزما در دانشآموزان به تفكيك جنسيت

\begin{tabular}{|c|c|c|c|c|c|}
\hline \multicolumn{6}{|c|}{ 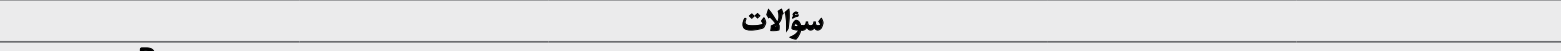 } \\
\hline $\begin{array}{c}\text { P } \\
\text { (Pearson Chi-square) }\end{array}$ & $17+*=\sqrt{5}$ & Sختر & 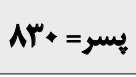 & & جنسيت \\
\hline \multicolumn{6}{|c|}{ آيا اين كودى شما تاكنون بثورات خارش دارى كه حداقل به ملت 8 ماه ظاهر و نايديد شود داشته است؟ } \\
\hline$+1+\infty 1$ & $r+1(1 r / 8)$ & $V A(9 / Y)$ & $\mid r g(1 \Delta / T)$ & تعلداد(دصرد) & بله \\
\hline \multicolumn{6}{|c|}{ "در r اماه كذشته آيا اين كودك شما بثورات خارشدار داشته است؟ } \\
\hline 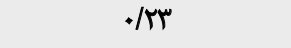 & $198(1+/ 4)$ & gه(N) & $1 \cdot 1(1 T / T)$ & 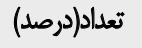 & بله \\
\hline \multicolumn{6}{|c|}{ 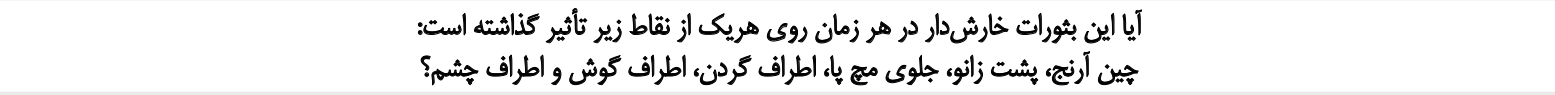 } \\
\hline \multirow[t]{2}{*}{$+/ 2$} & $r+\Delta(1 Y / \Lambda)$ & FA(N) & $\mid f *(\mid g / \lambda)$ & تعداد(درصد) & بله \\
\hline & \multicolumn{5}{|c|}{ بثورات خارشدار اولين بار در جه سنى در اين كودى شما ايجاد شده است؟ } \\
\hline \multirow{3}{*}{. $/ \Delta r$} & $9(\cdot 18)$ & $\Delta(\cdot / 8)$ & $f(* / \Delta)$ & & كمتر از ب سالكي \\
\hline & $r q(1 / 1)$ & $r \cdot(T / P)$ & $r q(r / \Delta)$ & تعداد(درصد) & 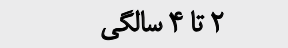 \\
\hline & $1 \cdot N(\varepsilon / \Lambda)$ & $P \cdot(\Delta / T)$ & EN(NY) & & 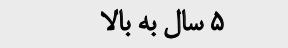 \\
\hline \multicolumn{6}{|c|}{ در rا ماه كذشته آيا اين بثورات به طور كامل مشخص و واضح بوده است؟ } \\
\hline \multirow[t]{2}{*}{. } & $\operatorname{lrg}(N \Delta)$ & $\Delta)(8 / 9)$ & $N \Delta(1 \cdot / Y)$ & ثعلاد(درصد) & بله \\
\hline & \multicolumn{4}{|c|}{ در rا ماه كذشته كودك شما جند بار به دليل اين بثورات از خواب شبانه بيدار شله است؟ } & \\
\hline \multirow{3}{*}{.$/ A 1$} & $\| \mu(V /)$ & $r \mu(\Delta / \mathcal{C})$ & $V+(N+)$ & & هركز بيدار نشده است \\
\hline & rA(T/Y) & $10(1 / 9)$ & $r(r / \Lambda)$ & تعلداد(دصرد) & يك شب در هفته \\
\hline & $10(\cdot / 9)$ & $V(\cdot / 9)$ & $\Lambda(1 / *)$ & & بيشتر از يك شب در هفته \\
\hline \multicolumn{6}{|c|}{ 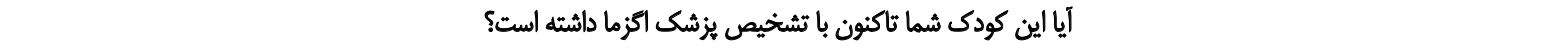 } \\
\hline.$/ N \Delta$ & $111(8 / 9)$ & $\Delta \Delta(Y / I)$ & $\Delta E(E / V)$ & ثعداد(دوصد) & بله \\
\hline
\end{tabular}

•هون كه بعضى از شركت كنندكان بيش از يك منطقه دركيرى را بيان كرده بودن تعداد از 198 نفر بيشتر شده است.

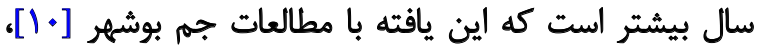

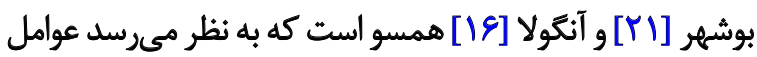
محيطى و تغذيهاي در اين رابطه تأثير كذار باشند.

خرويف يكى از متغيرهايى است كه هرجند در مطالعات اندكى إنى

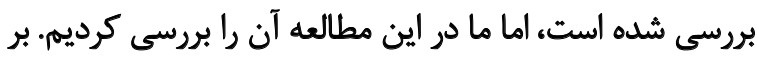

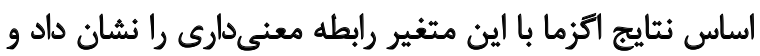

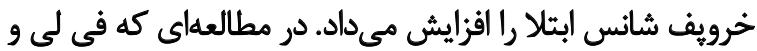

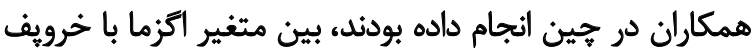

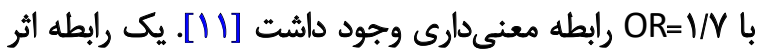

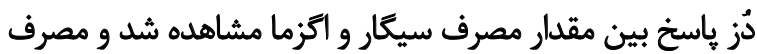

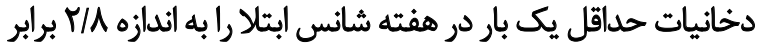

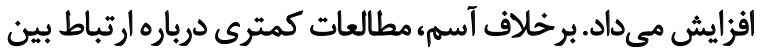
سيكار كشيدن و اكزما انجام شده است. مطالعات نشان دادئ دادند
يك شب در هفته و 9 درصد بيش از يك شب در هفته از خواب

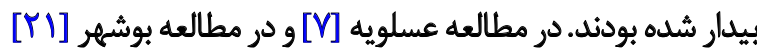

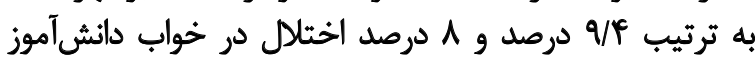

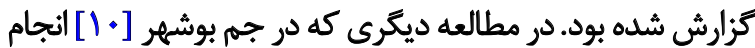

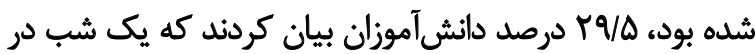

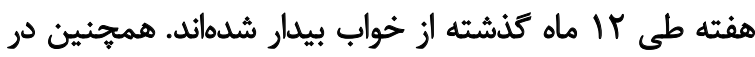

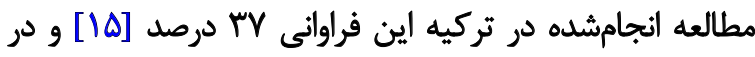

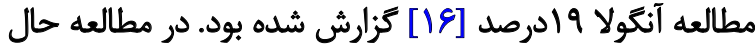

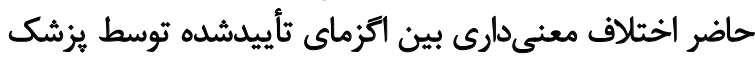

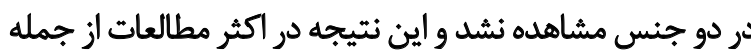

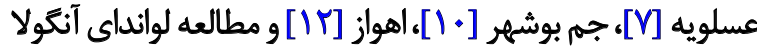

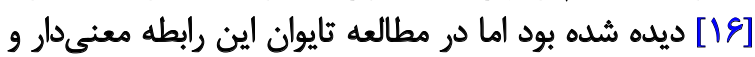

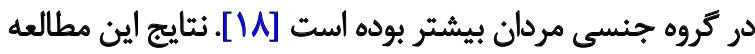

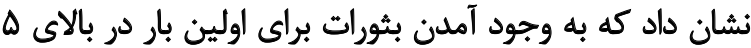


جدول r. ضرايب مربوط به متغيرهاي مستقل مرتبط با اتزما در دائشآموزان در مدل ركرسيون لجستيك جئدكانه

\begin{tabular}{|c|c|c|c|c|c|}
\hline $\mathbf{P}$ & $\begin{array}{l}\text { Adjusted Odds } \\
\text { Ratio (Cl) }\end{array}$ & $\mathbf{P}$ & $\begin{array}{l}\text { Crude Odds } \\
\text { Ratio (Cl) }\end{array}$ & \multicolumn{2}{|c|}{ متغيرهاى مستقل } \\
\hline $.10 . .1$ & $\begin{array}{c}r / r \Delta(1 / \Delta r-r / \Delta q) \\
1\end{array}$ & $.1 \ldots .1$ & $\begin{array}{c}T / Q(1 / F E-T / N E) \\
1\end{array}$ & ندارد & سابقه آلرزى در خانواده \\
\hline$+1+\ldots 1$ & $\begin{array}{c}r / \cdots(1 / Q \Lambda-r / \Delta \Delta) \\
1\end{array}$ & $+1+\ldots+1$ & $\begin{array}{c}r / T F(T / M \backslash-Q / I F) \\
1\end{array}$ & ندارد & خرويف يلر \\
\hline $.10 p^{p}$ & $\begin{array}{c}1 / P \cdot(1 / \cdot . r-r / \Delta A) \\
1\end{array}$ &.$/ . . r$ & $\begin{array}{c}V / U(1 / T F-r / \cdot V) \\
1\end{array}$ & ندارد & نكهبارى حيوان مر منزل \\
\hline .1 .1 &.$/ 1 \Delta(\cdot 181-. / 9 \Delta)$ & $.1 \cdot 1$ &.$/ N E(\cdot M r-1 / \cdot r)$ & كمى & 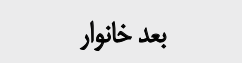 \\
\hline $\begin{array}{l}.19 \\
.101 \\
.1+r\end{array}$ & 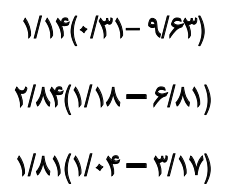 & $\begin{array}{l}. / n \\
.1+.1 \\
.1+.1\end{array}$ & 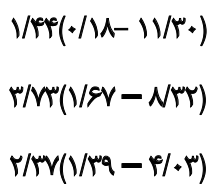 & 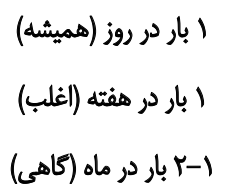 & مصرف دخانيات \\
\hline & 1 & & 1 & هركز & \\
\hline
\end{tabular}

متحده انجام شُه است، نشان مي دهد قرار كرفتن در معرض

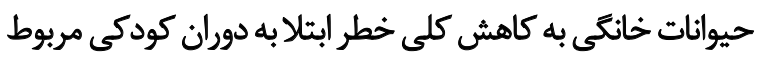

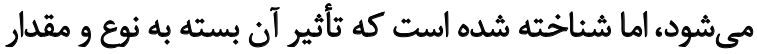

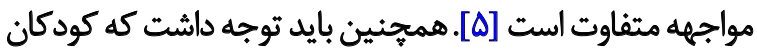

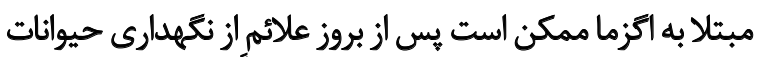

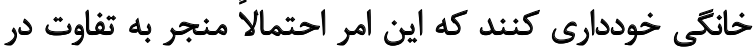

كه استعمال سيعار خطر اكزما رادر مطالعات جمم بوشهر [1.]

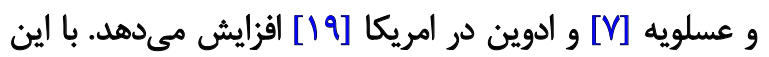

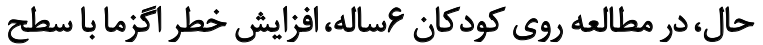

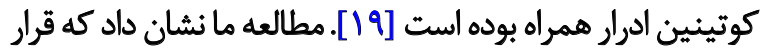

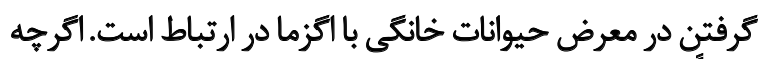

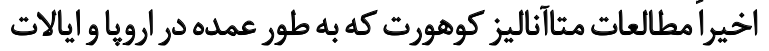

نسبت شانس و فاصله اطمينان

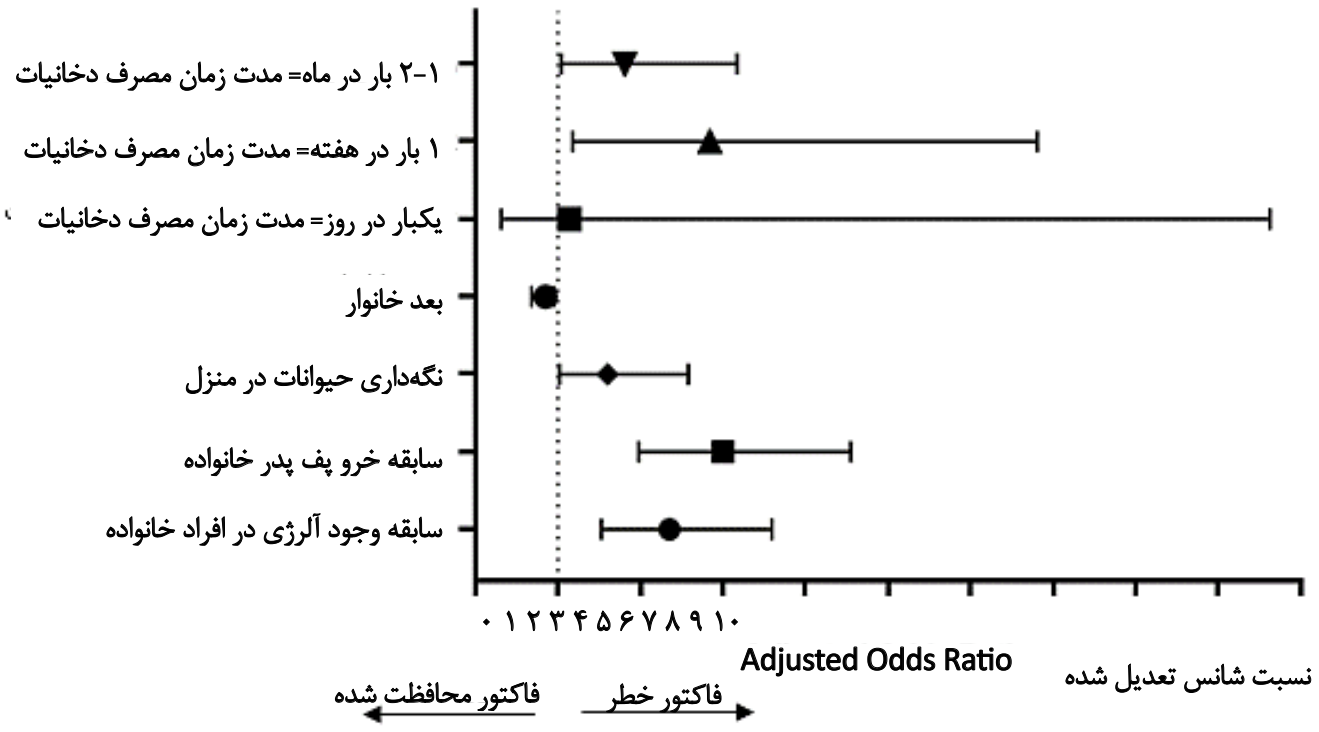

تصوير ا. نسبث شائس و فاصله اطميئان 


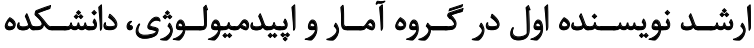

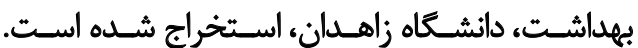

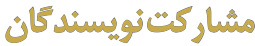

طراحى تحقيق، جمعآورى دادهها، يالايش دادهها، كزارش

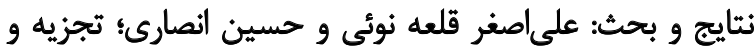

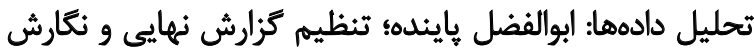

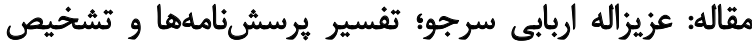

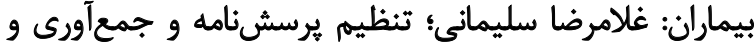
بازبينى دادههاى جمعآورىشده: على مشكينيان.

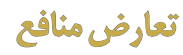

بدينوسيله نويسندگان تصريح مى كنئد كه هيجگَونه تضاد منافعى در برُوهش حاضر وجود ندارد.

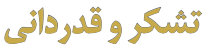

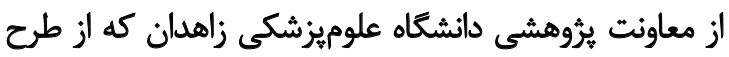
مذكور حمايت مالى كردند صميمانه تقدير و تشكر مى كنيهم.
يافتهها بين دورههاي داشتن حيوانات خانكى مي إشود. در مطالعه

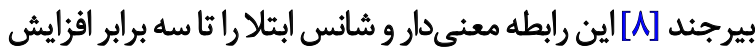

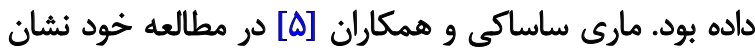

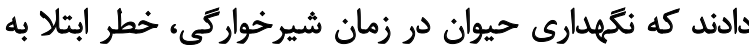

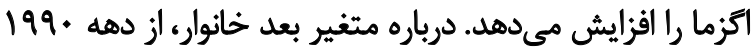

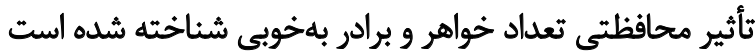

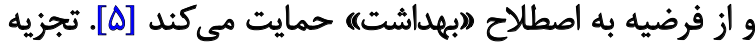

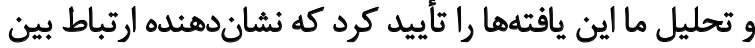

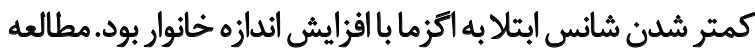

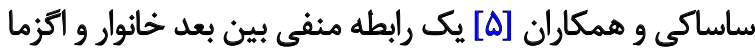

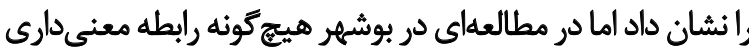

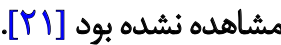

نقطه قوت مطالعه حاضر انجام يروهش در دانش آموزان تروه

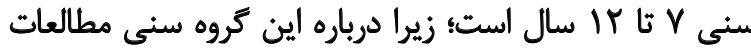

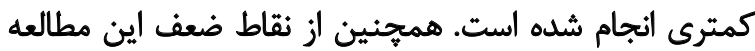
مي توان به همكارى نكردن بعضى والدين در اين يثوهش اشئ اشاره

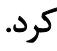

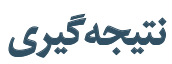

مطالعه حاضر شواهدى درباره ارتباط بين عوامل

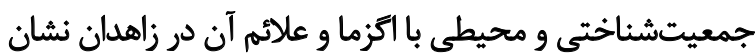

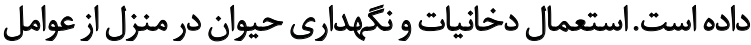

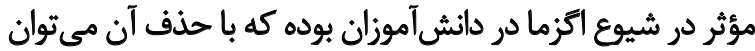

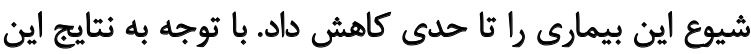

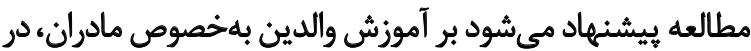
مورد توجه به اين فاكتورهاى مساعدكنئده تأكيد شود.

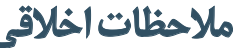

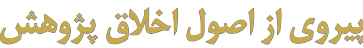

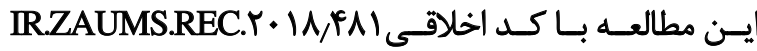

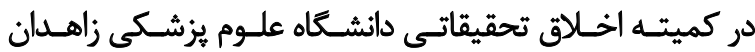

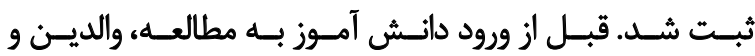

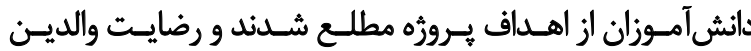

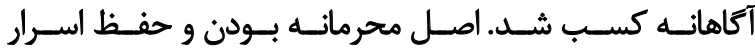

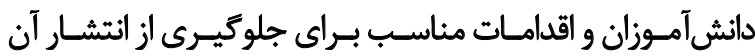

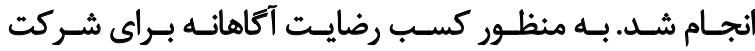

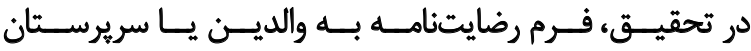

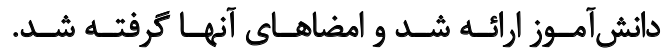

$$
\text { مأم }
$$

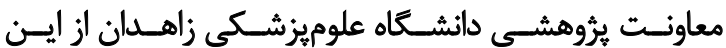

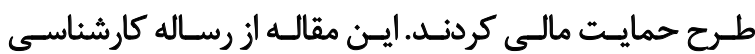




\section{References}

[1] Ferrandiz-Mont D, Wahyuniati N, Chen HJ, Mulyadi M, Zanaria TM, Ji DD. Hygiene practices: Are they protective factors for eczema symptoms? Immun Inflamm Dis. 2018; 6(2):297-306. [DOI:10.1002/iid3.217]

[2] Sathishkumar D, Moss C. Topical therapy in atopic dermatitis in children. Indian J Dermatol. 2016; 61(6):656-61. [DOI:10.4103/00195154.193677[ [PMCID] [PMID]

[3] Chan CWH, Wong RS, Law PTW, Wong CL, Tsui SKW, Tang WPY, et al. Environmental factors associated with altered gut microbiota in children with eczema: A systematic review. Int J Mol Sci. 2016; 17(7):1147. [DOI:10.3390/ijms17071147]

[4] Versini M, Jeandel PY, Bashi T, Bizzaro G, Blank M, Shoenfeld Y. Unraveling the hygiene hypothesis of helminthes and autoimmunity: Origins, pathophysiology, and clinical applications. BMC Med. 2015; 13:81. https://link.springer.com/article/10.1186/s12916-015-0306-7

[5] Sasaki M, Yoshida K, Adachi Y, Furukawa M, Itazawa T, Odajima H, et al. Environmental factors associated with childhood eczema: Findings from a national web-based survey. Allergol Int. 2016; 65(4):420-4. [DOI:10.1016/j.alit.2016.03.007]

[6] Hajavi J, Tolide-ie HR, Rastgoie Chavoshlu S, Salehi Rezve M, Modoodi Yaghooti M, Rahimi J. [DO rural and urban children have different prevalence of allergic disorders in Gonabad? (Persian)] Horizon Med Sci. 2012; 18(2):21-6. http://hms.gmu.ac.ir/article-1-1452-en.html

[7] Gooya M, Shirkani A, Tahmasebi R, Omrani A, Gheybi MK, Darabi H, et al. [Prevalence of asthma and allergic diseases and its risk factors in school children aged (6-7 and 13-14 years) in Assalouyeh city, Bushehr province based on III ISAAC protocol phase I, in 2014 (Persian)]. Iran South Med J. 2017; 20(1):57-69. [DOI:10.18869/acadpub.ismj.20.1.57]

[8] Ghaderi R, Tabiee S, Peyrovi S, Jafari Pour M. [Prevalence of atopic dermatitis and its risk factors in 2-5 years old children at kindergartens of Birjand city (2008) (Persian)]. J Birjand Univ Med Sci. 2012; 19(3):286-93. http://journal.bums.ac.ir/article-1-1115-en.html

[9] Karimi M, Mirzaei M, Ahmadieh MH. [Prevalence of asthma, allergic rhinitis and eczema symptoms among 13-14 year-old school children in Yazd in 2003 (Persian)]. Jundishapur Sci Med J. 2007; 6(3):270-5. https:// www.sid.ir/fa/journal/ViewPaper.aspx?ID=72329

[10] Assadi T, Gheybi M, Shirkani A, Movahed A, Khoddami S, Ashourinejad $A$, et al. [Study of prevalence and risk factors of asthma and allergic diseases among school children (6-7 and 13-14 years) based on ISAAC protocol in Jam City, Bushehr province in 2014 (Persian)]. Iran South Med J. 2017; 19(6):972-81. [DOI:10.18869/acadpub.ismj.19.6.972]

[11] Li F, Zhou Y, Li S, Jiang F, Jin X, Yan C, et al. Prevalence and risk factors of childhood allergic diseases in eight metropolitan cities in China: A multicenter study. BMC Public Health. 2011; 11:437. [DOI:10.1186/14712458-11-437]

[12] Shakurnia AH, Assar S, Afra M, Latifi M. Prevalence of asthma among schoolchildren in Ahvaz, Islamic Republic of Iran. East Mediterr Health J. 2010; 16(6):651-6. https://apps.who.int/iris/handle/10665/117934

[13] Ghaffari J, Aarabi M. The prevalence of pediatric asthma in the Islamic Republic of Iran: A systematic review and meta-analysis. J Pediatr Rev. 2013; 1(1):2-11. http://jpr.mazums.ac.ir/article-1-31-en.html

[14] von Kobyletzki LB, Bornehag CG, Hasselgren M, Larsson M, Lindström $\mathrm{CB}$, Svensson Å. Eczema in early childhood is strongly associated with the development of asthma and rhinitis in a prospective cohort. BMC Dermatol. 2012; 12:11. [DOI:10.1186/1471-5945-12-11]
[15] Topal E, Kaplan F, Turker K, Kutluturk K, Gozukara Bag H. [The prevalence of allergic diseases and associated risk factors in the 6-7 age children who are living in Malatya, Turkey (Turkish)]. Asthma Allergy Immunol. 2017; 15:129-34. http://abakus.inonu.edu.tr/xmlui/handle/11616/13496

[16] Arrais M, Lulua O, Quifica F, Rosado-Pinto J, Gama JMR, Taborda-Barata L. Prevalence of asthma, allergic rhinitis and eczema in 6-7-year-old schoolchildren from Luanda, Angola. Allergol Immunopathol (Madr). 2019; 47(6):523-34. [DOI:10.1016/j.aller.2018.12.002]

[17] Bolat E, Arikoglu T, Sungur MA, Batmaz SB, Kuyucu S. Prevalence and risk factors for wheezing and allergic diseases in preschool children: $A$ perspective from the Mediterranean coast of Turkey. Allergol Immunopathol (Madr). 2017; 45(4):362-8. [DOI:10.1016/j.aller.2017.01.002]

[18] Hwang CY, Chen YJ, Lin MW, Chen TJ, Chu SY, Chen CC, et al. Prevalence of atopic dermatitis, allergic rhinitis and asthma in Taiwan: a national study 2000 to 2007. Acta Derm Venereol. 2010; 90:589-94. [DOI:10.2340/00015555-0963]

[19] Mitchell EA, Beasley R, Keil U, Montefort S, Odhiambo J, ISAAC Phase Three Study Group. The association between tobacco and the risk of asthma, rhinoconjunctivitis and eczema in children and adolescents: Analyses from Phase Three of the ISAAC programme. Thorax. 2012; 67:941-9. [Doi:10.1136/thoraxjnl-2011-200901]

[20] Farajzadeh S, Esfandiarpour I, Sedaghatmanesh M, Saviz M. Epidemiology and clinical features of atopic dermatitis in Kerman, a desert area of Iran. Ann Dermatol. 2014; 26(1):26-34. [DOI:10.5021/ad.2014.26.1.26]

[21] Farrokhi S, Gheybi MK, Movahhed A, Dehdari R, Gooya M, Keshvari S, et al. Prevalence and risk factors of asthma and allergic diseases in primary schoolchildren living in Bushehr, Iran: Phase I, III ISAAC protocol. Iran J Allergy Asthma Immunol. 2014; 13(5):348-55. https://ijaai.tums.ac.ir/ index.php/ijaai/article/view/434 